\title{
Wie Ihr Praxisteam reibungsloser arbeiten kann
}

\author{
Dieser MMW-Ausgabe liegt wieder „info praxisteam“ bei, das Magazin für Medizinische Fachange- \\ stellte. Das Hauptthema in Heft 4/16 ist die Teamarbeit in der Arztpraxis. Mit der richtigen Einstellung \\ und einigen Tipps könnte es bei Ihnen bald so rund laufen wie bei Manuel Neuer und Co.
}

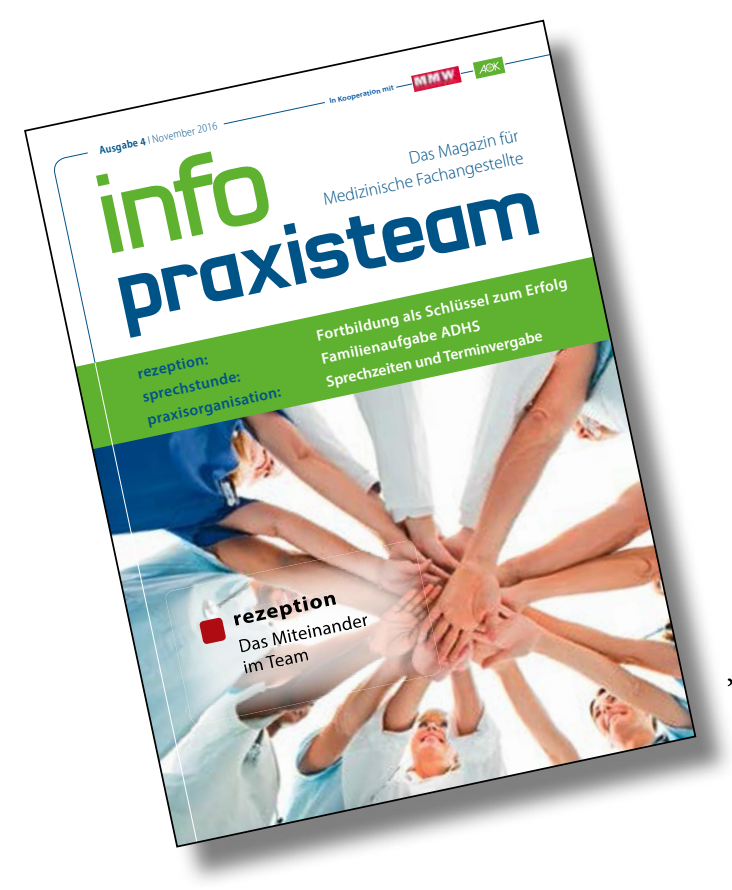

_ Verbindliche Strukturen sind eine wichtige Voraussetzung für erfolgreiche Teamarbeit. Eine basisdemokratische Entscheidungsfindung kann in einer Hausarztpraxis nämlich nicht funktionieren; klare Regelungen sind zwingend erforderlich. Mindestens genauso wichtig ist jedoch die "Interaktionsebene“. Die persönlichen Seiten der Teammitglieder haben großen Einfluss auf die Rolle im Team und damit auch auf die Zusammenarbeit.

Bei einer Fußballmannschaft lässt sich gut beobachten, dass verschiedene Talente den Erfolg eines Teams ausmachen - wenn man sie auch entsprechend ihrem Können einsetzt. Deshalb steht in der Nationalmannschaft Manuel Neuer im Tor, Jérôme Boateng in der Abwehr und Thomas Müller im Sturm - und weder Müller im Tor noch Boateng im Sturm.

„info praxisteam“ beschäftigt sich auch mit den wissenschaftlichen Grund- lagen, die in den späten 1970er-Jahren der britische Psychologieprofessor Meredith Beibin lieferte. Er hatte in seinen Seminaren ein interessantes Phänomen entdeckt: Zu einer Gruppenarbeit hatten sich die intelligentesten Kursteilnehmer zu einem Team zusammengefunden und dieses Team lieferte dann das schlechteste Ergebnis.

Beibin beschrieb daraufhin Teamrollen als ,eine Tendenz, sich gegenüber anderen auf eine besondere Art zu verhalten, einen Beitrag zu leisten und mit ihnen zusammen zu wirken“. Demnach verfügt jeder Mensch über Stärken und „erlaubte Schwächen“, die er bestimmten Rollen zuordnet, von denen Beibin neun beschreibt.

\section{Aus Konflikten im Team sollte man keine große Sache machen}

Doch was bringt es in der Praxis, Menschen einzelnen Kategorien zuzuordnen? Kaum ein Praxisteam zählt neun Köpfe - da ist es schon theoretisch kaum machbar, die Rollen nach der Theorie zu verteilen. Dennoch bringt das Wissen um die Rollenverteilung im Team eine ganze Menge. Denn sich in ein Team einzubringen heißt auch, die eigenen Bedürfnisse nicht zu ignorieren. Sind alle auf dem Egotrip, leidet die Teamleistung deutlich, weil zu viel Zeit und Energie auf Machtkämpfe verwendet werden. Andererseits ist es auch nicht gut, sich ständig zurückzunehmen. Nur wer an sich selbst glaubt, kann andere überzeugen und so dem Team helfen.

Und wenn dabei mal die Interessen der einzelnen Teammitgleider aufeinander prallen, sollte das keine große Sache sein. Mal gibt der Eine nach, mal der Andere, und oft kann man sich einfach in der Mitte treffen - der klassische Kompromiss. Das ist für viele so selbstver- ständlich, dass wir es kaum noch bewusst wahrnehmen. Schwierig wird es immer nur dann, wenn existierende Interessenkonflikte nicht angesprochen werden. Schnell entsteht dann ein Teufelskreis, der mit dem eigentlichen Konfliktthema gar nichts mehr zu tun hat.

Klare Spielregeln helfen, Konflikte erst gar nicht entstehen zu lassen. So sind regelmäßige Teambesprechungen die beste Garantie dafür, dass keine wichtigen Informationen versteckt werden. Und dass niemand schlecht über Kollegen redet, sollte überall selbstverständlich sein.

In der Rubrik „sprechstunde“ geht es um ADHS bei Kindern und Jugendlichen als „Familienaufgabe“. In der „praxisorganisation“ gibt es Tipps zur Terminvergabe.

Dr. Reinhard Merz

\section{„info praxisteam“ im Web}

Unser MFA-Magazin erscheint viermal jährlich. Alle Beiträge sind unter

\section{www.info-praxisteam.de}

auch im Internet abrufbar und können direkt kommentiert werden.

Auch für mobile Endgeräte wie Smartphones oder Tablets ist „info praxisteam“ verfügbar. Die App wird sowohl für iOS von Apple als auch für Geräte mit Android-Plattform angeboten. Mehr darüber, wie man die App beziehen kann, erfahren Sie auf der oben angegebenen Website.

Zu den Beiträgen im Heft gibt es von der Redaktion ausgesuchte Verweise auf weiterführende Informationen online. Viele davon sind auch für Sie als Arzt interessant.

Sie erreichen uns per E-Mail unter:

redaktion@info-praxisteam.de 